\title{
COVID-19 in Pediatric Survivors of Childhood Cancer and Hematopoietic Cell Transplantation from A Single-Center in New York City
}

\author{
Lauren Kurlander ${ }^{1}$, Zoltan Antal ${ }^{1}$, Amelia DeRosa ${ }^{2}$, Deborah Diotallevi ${ }^{3}$, Elaine \\ Pottenger $^{1}$, Nadia Wilson ${ }^{1}$, Stacie Corcoran ${ }^{1}$, Farid Boulad ${ }^{1}$, and Danielle Novetsky \\ Friedman ${ }^{2}$ \\ ${ }^{1}$ Memorial Sloan Kettering Cancer Center \\ ${ }^{2}$ Memorial Sloan Kettering \\ ${ }^{3}$ Memorial Sloan-Kettering Cancer Center
}

October 29, 2020

\begin{abstract}
Childhood cancer survivors are at increased risk for treatment-related late effects; data are lacking on how COVID-19 infection impacts this cohort. We assessed COVID-19-related symptoms; SARS-CoV-2 IgG seroprevalence; and rate of COVID-19-related hospitalization among 321 asymptomatic survivors of childhood cancer or transplantation seen for routine long-term follow-up between May-September 2020 in a New York City tertiary cancer center. While $11 \%(\mathrm{n}=35)$ reported possible COVID-19related symptoms, $7.8 \%(\mathrm{n}=20)$ of those tested had positive SARS-CoV-2 IgG, and only 1 patient (0.3\%) required COVID-19 related hospitalization. This report suggests that childhood cancer survivors are at low risk for COVID-19 complications.
\end{abstract}

\section{INTRODUCTION}

The coronavirus disease-2019 (COVID-19) pandemic has unique implications for the growing cohort of survivors of childhood cancer and hematopoietic cell transplantation (HCT) for non-malignant hematologic/immune disorders, ${ }^{1,2}$ referred to herein as "survivors." Investigators have postulated that history of underlying immune dysfunction and/or organ injury due to prior therapeutic exposures increase survivors' risk of contracting severe acute respiratory syndrome coronavirus-2 (SARS-CoV-2) and/or increased severity of COVID-19..$^{3,4,5}$ Using the Childhood Cancer Survivor Study (CCSS) cohort, Perkins et. al previously found an increased incidence of overall infections and a higher risk of infection-related mortality among survivors of childhood cancer as compared to unaffected siblings for at least 35 years after therapy. ${ }^{6}$ Whether these infectious risk findings can be applied to the COVID-19 pandemic and inform guidelines for childhood cancer survivors as they return to school or work is unknown. ${ }^{5}$ In an effort to fill this gap, we assessed reported COVID-19 symptoms, exposures, and/or hospitalization, as well as SARS-CoV-2 IgG status, in a cohort of pediatric survivors presenting for routine long-term follow-up (LTFU) either in-person or virtually in the early months of the COVID-19 pandemic in New York City, one of the original epicenters of the pandemic.

\section{METHODS}

This retrospective review included all consecutive survivors seen in Memorial Sloan Kettering (MSK)'s Pediatric LTFU Clinic between May 5-September 10, 2020, which provides risk-based care to survivors of childhood cancer and HCT diagnosed at age [?]18 who are [?]1-year off-therapy at program entry. 
All patients were scheduled for routine LTFU (in-person or via telehealth) during this interval; SARS-CoV-2 IgG testing was offered when venipuncture was being performed for clinical indications. ${ }^{7,8}$ During all visits, patients were assessed for known COVID-19 exposure(s) after March 1, 2020; COVID-19 related symptoms [fever, cough, respiratory distress, loss of smell/taste]; and COVID-19-related hospitalization. A subset of patients had SARS-CoV-2 PCR testing performed locally or on-site if they had traveled from high-prevalence areas, reported active symptoms of infection, or required testing for preprocedural indications. ${ }^{9}$

The protocol was approved by the MSK Institutional Review/Privacy Board.

\section{RESULTS}

Table 1 summarizes demographics of 321 unique childhood cancer survivors seen during this interval; 227 (70.7\%) in-person and $94(29.3 \%)$ via telehealth. Survivors were 1 - 18.6-years (median 6.9 years) after completion of all cytotoxic therapies. Most common diagnoses included leukemia/lymphoma $(28.4 \%, \mathrm{n}=91)$, neuroblastoma $(19 \%, \mathrm{n}=61)$, and non-malignant hematologic disorders $(12.5 \%, \mathrm{n}=40)$. Ninety-nine patients $(30.9 \%)$ had history of prior HCT. Thirty-five patients (10.9\%) reported prior symptoms consistent with COVID-19 infection. SARS-CoV-2 serology results in the context of reported symptoms and known COVID19 exposures are summarized in Table $\mathbf{2}$. Six patients (1.9\%) experienced family member deaths due to COVID-19, including grandparents $(\mathrm{n}=5)$ and one aunt.

Among 257 (80.1\%) patients who had SARS-CoV-2 IgG testing, 20 (7.8\%) patients had positive antibodies [Table 1 ]. Most common diagnoses among patients with positive serology included leukemia/lymphoma $(25 \%, \mathrm{n}=5)$, non-malignant hematologic disorders $(25 \%, \mathrm{n}=5)$, and sarcoma $(15 \%, \mathrm{n}=3)$. One-half of patients with positive SARS-CoV-2 IgG resided in two zip codes located in Kings County, Brooklyn $(30 \%, \mathrm{n}=6)$ and the Bronx $(20 \%, \mathrm{n}=4)$. Of all patients seen, these residencies were reported by $14.6 \%(\mathrm{n}=47)$ and $3.7 \%$ $(\mathrm{n}=12)$, respectively. Among patients with positive SARS-CoV-2 serology, 35\% ( $\mathrm{n}=7)$ were HCT survivors.

Five $(1.6 \%)$ patients reported history of PCR-confirmed SARS-CoV-2 infection. Four of 5 patients had COVID-19 antibodies drawn at their visits, and all had positive seroconversion between 2 to 5 months after reported infection (100\%). Among these patients, 2 had never developed symptoms; 2 had mild symptoms including fever $(n=1)$, headache $(n=1)$, and loss of sense of smell $(n=2)$; and $1(0.3 \%)$ required hospitalization for respiratory insufficiency and myocarditis with subsequent full recovery. None of our patients developed multisystem inflammatory syndrome in children (MIS-C).

\section{DISCUSSION}

In this large pediatric survivor cohort followed in New York City (NYC), one of the earliest epicenters of the pandemic, we found a low prevalence of reported COVID-19-related symptoms or hospitalization. While $7.8 \%$ of those tested had positive SARS-CoV-2 IgG and probable prior infection, only one patient $(0.3 \%)$ required medical evaluation and subsequent hospitalization. Patients residing in just two zip codes, which were known COVID-19 "hot spots," ${ }^{10}$ made up 50\% of patients with positive SARS-CoV-2 serology. This suggests that living in high prevalence areas of SARS-CoV-2 may put survivors at increased risk of contracting the virus.

This cohort's relatively low disease burden is consistent with other reports from oncology settings in which patients on active treatment appear to be at similar risk as non-oncology populations for COVID-19..$^{9,11,12}$ Interestingly, $7.8 \%$ of our tested cohort had positive SARS-CoV-2 antibodies, compared to $29.9 \%$ of individuals aged 0-17 in New York City. ${ }^{10}$ We hypothesize that survivors and their families were already adept at mask-wearing and social distancing and thus less likely to contract disease. Alternatively, a subset of survivors may have been unable to mount a serologic response to COVID-19 due to impaired immunity related to prior underlying diagnosis or cytotoxic treatment(s). Our patient population is also less racially and ethnically diverse than the larger New York City pediatric population and may partially accounts for the relatively low disease burden in this cohort. Future investigation is necessary to clarify these findings.

Various limitations must be considered. While the clinical significance of SARS-CoV-2 serology remains unclear, positive antibodies appear to be a reasonable indicator of prior disease in asymptomatic individuals..$^{8,13}$ 
Additionally, since only one patient reported severe disease requiring hospitalization, we were unable to assess associations between prior cancer therapies and COVID-19 severity. The retrospective, single-center design and reliance on patient/family recall also likely introduced bias. However, since nearly one-third of visits were conducted via telehealth, the cohort includes patients who were unable/unwilling to travel to NYC and individuals who were too ill to attend in-person visits.

Our preliminary data suggest that pediatric survivors do not experience a high burden of COVID-19-related complications. Based on currently available data, childhood cancer survivors should follow national precautions for mask-wearing, social distancing, and hand hygiene as the country prepares for a potential upcoming COVID-19 resurgence. Longitudinal follow-up of large survivor cohorts will be required to analyze the impact of treatment exposures and existing co-morbidities on COVID-19 severity and inform guideline development for this high-risk population.

Conflict of Interest Statement: Danielle Novetsky Friedman held an advisory role for Fennec Pharmaceuticals in the past 36 months.

Acknowledgements : This study was supported by the Memorial Sloan Kettering Core Grant (P30 CA008748) and the Meg Berte Owen Foundation. The NIH and the Meg Berte Owen Foundation had no role in the design and conduct of the study.

\section{REFERENCES}

1. Phillips SM, Padgett LS, Leisenring WM, et al. Survivors of childhood cancer in the United States: prevalence and burden of morbidity. Cancer Epidemiol Biomarkers Prev . 2016;24(4):653-663.

2. Cheung AT, Li WHC, Ho LLK, Chan GCF, Chung JOK. Health support to pediatric cancer survivors and their families during the COVID-19 pandemic [published online ahead of print, 2020 Aug 9]. Pediatr Blood Cancer. 2020;e28441.

3. Nekhlyudov L, Duijts S, Hudson SV, et al. Addressing the needs of cancer survivors during the COVID-19 pandemic. J Cancer Surviv. 2020;14(5):601-606.

4. Fung M, Babik JM. COVID-19 in Immunocompromised Hosts: What We Know So Far [published online ahead of print, 2020 Jun 27]. Clin Infect Dis. 2020;ciaa863.

5. Verbruggen LC, Wang Y, Armenian SH, et al. Guidance regarding COVID-19 for survivors of childhood, adolescent, and young adult cancer: A statement from the International Late Effects of Childhood Cancer Guideline Harmonization Group. Pediatr Blood Cancer . 2020;67(12):e28702.

6. Perkins JL, Chen Y, Harris A, et al. Infections among long-term survivors of childhood and adolescent cancer: a report from the Childhood Cancer Survivor Study. Cancer . 2014;120(16):2514-2521.

7. Bryan A, Pepper G, Wener MH, et al. Performance Characteristics of the Abbott Architect SARSCoV-2 IgG Assay and Seroprevalence in Boise, Idaho. J Clin Microbiol . 2020;58(8).

8. Deeks JJ, Dinnes J, Takwoingi Y, et al. Antibody tests for identification of current and past infection with SARS-CoV-2. Cochrane Database Syst Rev . 2020;6(6):CD013652.

9. Boulad F, Kamboj M, Bouvier N, Mauguen A, Kung AL. COVID-19 in Children With Cancer in New York City. JAMA Oncol.2020:e202028.

10. NYC Department of Health. Coronavirus disease 2019 (COVID-19) Data. 2020; https://www1.nyc.gov/site/doh/covid/covid-19-data.page. Accessed September 18, 2020.

11. de Rojas T, Pérez-Martínez A, Cela E, Baragaño M, Galán V, Mata C, Peretó A, Madero L. COVID-19 infection in children and adolescents with cancer in Madrid. Pediatr Blood Cancer. 2020 Jul;67(7):e28397.

12. Fuereder T, Berghoff AS, Heller G, et al. SARS-CoV-2 seroprevalence in oncology healthcare professionals and patients with cancer at a tertiary care centre during the COVID-19 pandemic. ESMO Open . 2020;5(5):e000889.

13. Sethuraman N, Jeremiah SS, Ryo A. Interpreting Diagnostic Tests for SARS-CoV-2. JAMA. 2020;323(22):2249-2251.

\section{Hosted file}


LTFU COVID brief report_Table 1.pdf available at https://authorea.com/users/371254/articles/ 489657-covid-19-in-pediatric-survivors-of-childhood-cancer-and-hematopoietic-celltransplantation-from-a-single-center-in-new-york-city

\section{Hosted file}

LTFU COVID brief report_Table 2.pdf available at https://authorea.com/users/371254/articles/ 489657-covid-19-in-pediatric-survivors-of-childhood-cancer-and-hematopoietic-celltransplantation-from-a-single-center-in-new-york-city 